\title{
Unstructured, curved elements for the two-dimensional high order discontinuous control-volume/finite-element method
}

\author{
By G. Stipcich and M. Piller
}

This is the pre-peer reviewed version of the following article:

"Unstructured, curved elements for the two-dimensional high order discontinuous control-volume/finite-element method", International Journal for Numerical

Methods in Engineering, Volume 102, Issue 1, pages 22-43, 6 April 2015 , which has been published in final form at

http://onlinelibrary.wiley.com/doi/10.1002/nme.4844/abstract 


\section{Unstructured, curved elements for the two-dimensional high order discontinuous control-volume/finite-element method}

\begin{tabular}{|r|l|}
\hline Journal: & International Journal for Numerical Methods in Engineering \\
\hline Manuscript ID: & Draft \\
\hline Wiley - Manuscript type: & Research Article \\
\hline Date Submitted by the Author: & n/a \\
\hline Complete List of Authors: & $\begin{array}{l}\text { Stipcich, Goran; Basque Center for Applied Mathematics, } \\
\text { Piller, M; University of Trieste, Dipartimento di Ingegneria e Architettura }\end{array}$ \\
\hline Keywords: & $\begin{array}{l}\text { Spectral, Finite volume methods, Curved, Discontinuous, Finite element } \\
\text { methods, Mapping }\end{array}$ \\
\hline \multicolumn{2}{|l}{} \\
\hline
\end{tabular}




\title{
Unstructured, curved elements for the two-dimensional high order discontinuous control-volume/finite-element method
}

\author{
G. Stipcich*†, M. Piller ${ }^{\dagger}$ \\ BCAM - Basque Center for Applied Mathematics, Mazarredo 14, E48009 Bilbao, Basque Country - Spain
}

\section{SUMMARY}

Quadrilateral and triangular elements with curved edges are developed in the framework of spectral, discontinuous, hybrid control-volume/finite-element method for elliptic problems. In order to accommodate hybrid meshes, encompassing both triangular and quadrilateral elements, one single mapping is used. The scheme is applied to two-dimensional problems with discontinuous, anisotropic diffusion coefficients and the exponential convergence of the method is verified in the presence of curved geometries. Copyright $(\mathcal{C}$ 2014 John Wiley \& Sons, Ltd.

Received: June 4, 2014

KEY WORDS: Discontinuous method; Curved elements; Finite volume; Finite element; Hybrid; Spectral method; Mapping

\section{INTRODUCTION}

The control volume finite element method (CVFEM), also known as vertex- or node-based finite volume method, combines the inherent local numerical conservation property of the control volume method with the geometrical flexibility of the finite element method. The CVFEM can be seen as a finite-element method where volume indicator distributions are used as weight functions [1]. The CVFEM has been introduced by [2] for advection-diffusion problems on triangular elements, where the integral conservation equations are enforced on polygonal control volumes built around each node of the mesh. The method has been applied to the solution of the Poisson equation using quadrilateral elements by [3] and successively extended to advection-diffusion problems [4]. A CVFEM with bilinear, quadrilateral elements is proposed in [5]. Tetrahedral elements for incompressible flows are introduced by [6, 7, 8]. A detailed analysis of consistent and lumped versions of the CVFEM algorithm for diffusion-type problems, investigating the properties of bilinear, quadrilateral elements, nine-node (Lagrange) quadrilateral elements, eightnode Serendipity elements and six-node triangular elements is carried out by [9]. The author points

\footnotetext{
*Correspondence to: BCAM - Basque Center for Applied Mathematics, Mazarredo 14, E48009 Bilbao, Spain

$\dagger$ Email: gstipcich@bcamath.org URL: http://www.bcamath.org/en/people/stipcich

Università degli Studi di Trieste, Dipartimento di Ingegneria e Architettura, via A. Valerio 6/1, 34127 Trieste, Italy

Email: piller@units.it 


\section{NOMENCLATURE}

$\begin{array}{ll}\text { Symbols } \\ \mathbf{D} & \text { Diffusivity tensor } \\ d & \text { Number of dimensions } \\ e & \text { Generic element } \\ \widehat{e} & \text { Canonical element }[-1,1]^{d} \\ \widehat{e}_{t} & \text { Triangular canonical element } \\ h & \text { Mesh size } \\ \mathbf{I} & \text { Identity matrix } \\ i, j & \text { Dummy indices } \\ \mathbf{J}_{\mathbf{x}} & \text { Jacobian of the elemental mapping } \\ L & \text { Lagrange coefficient } \\ \mathcal{M}_{e} & \text { Elemental mapping } \\ \mathbf{M}^{e} & \text { Elemental mass matrix } \\ \mathbf{n} & \text { Unit normal vector } \\ P^{e} & \text { Element's polynomial order } \\ \mathbf{q} & \text { Flux vector } \\ Q & \text { Source term } \\ \mathbf{S} & \text { Element's side } \\ \mathcal{T}_{h} & \text { Triangulation of the domain } \\ u & \text { Scalar quantity } \\ & \end{array}$

V Control volume

$v_{h} \quad$ Piecewise-polynomial functions

$\mathbf{x} \quad$ Position vector

$X_{h} \quad$ Finite-dimensional subspace

X Coordinates of a point

Greek letters

$\delta_{i j} \quad$ Kronecker delta

$\eta \quad$ Position vector in $\widehat{e}$

$\Gamma_{D, N} \quad$ Dirichlet and Neumann boundary conditions

$\kappa \quad$ Condition number

$\Omega \quad$ Computational domain

$\phi_{i} \quad$ Basis functions

$\xi \quad$ Position vector in $\widehat{e}_{t}$

Abbreviations

d.o.f. Degrees of freedom

Other

$\sim \quad$ Numerical trace

- Geometrical entity in $\widehat{e}$

out some of the positive characteristics of the CVFEM approach: local conservation is achieved at control volume level and the discrete maximum principle is preserved [10].

In recent years, numerical methods with spectral convergence have been widely used in computational fluid dynamics (CFD) for solving flows with complex features. A high order CVFEM for unstructured grids was proposed by [11] for advection-diffusion problems, featuring a quadrature-free approach on quadrilateral elements. It is shown that an appropriate distribution of interpolation points and control-volume edges leads to a well conditioned matrix. The algorithm proposed in the present work is a discontinuous control-volume/finite-element method (DCVFEM). Discontinuous methods have been developing at first in the finite-element framework. The resulting discontinuous Galerkin methods (DGFEM) are characterized by the relaxation of the continuity constraint between neighboring elements, which is imposed in weak form through the so called numerical fluxes or numerical traces [12,13]. The specific formulation of numerical fluxes strongly affects the consistency, stability and accuracy of the method [12]. The discontinuous philosophy has been applied within the CVFEM framework [14, 15], giving rise to discontinuous control volume/finite element methods. Research in this area has been mainly focused on hyperbolic problems $[14,15]$. A thorough comparison of spectral GFEM, Taylor-Galerkin Least Square finite 
elements (TGLS), DGFEM and DCVFEM for the linear advection equation, using quadrilateral elements with interpolating polynomials of degree four to nine is provided by [15].

The SV method is a high-order, discontinuous, finite volume method, originally developed by [16] for hyperbolic conservation laws on two-dimensional, triangular, unstructured grids [16, 17, $18,19,20,21]$. Since then, the SV method has been successfully applied to the solution of the Euler [19] and Maxwell [21] equations. The SV method shares several features with the DCVFEM, such as discontinuous solution space, compact support for the approximations, $h-p$ refinement capability. The major difference between the SV and DCVFEM lies in the definition of the unknown degrees of freedom, which for SV are control-volume-averaged values, while in DCVFEM are nodal values. In addition, DCVFEM approximations of conservation laws require both volume and surface integrations, while SV requires surface integrations followed by a deconvolution procedure, used to derive point values from control volume averaged values [16]. Another significant difference can be identified in the kind of tessellations used to partition the primary elements of the mesh into control volumes, which are intended as a dual mesh with respect to the primary one. In the SV approach the simplex elements (triangles in 2D, tetrahedrons in 3D) are further subdivided into a structured pattern of subcells [22], whose number and shape depend on the degree of polynomial reconstruction. SV approximation with orders higher than three are based on rather complex subdivision patterns and reconstruction coefficients [19].

To the authors' best knowledge, so far DCVFEM has been proposed only with bar and quadrilateral elements $[15,23]$, as these types of elements may be subdivided very naturally into an arbitrary number of control volumes, thus achieving high order of accuracy. The present work provides a two-dimensional extension of the spectral DCVFEM method to diffusion-dominated problems on unstructured, hybrid meshes composed of quadrilaterals and triangles with curved edges. The mixed formulation is used, where both the transported scalar field and the associated diffusive flux are considered as dependent quantities [24, ch. 11, 12]. The mixed formulation allows to achieve an accurate approximation of both the scalar quantity and the corresponding flux, to relax the regularity requirements on the dependent variables [25]. Integrating both the conservation and the constitutive equations over control volumes, which are entirely contained within elements, gives rise to inter-element fluxes, which, in the present work, are represented by the LDG formulation [26]. Both quadrilateral and triangular elements are mapped onto the standard square $[-1,1]^{2}$, with the advantage of using the same assembly procedure. For triangular elements the collapsed coordinate mapping is used [27], allowing for a straightforward and intuitive subdivision of the primary element into control volumes for any (high) order of accuracy. The high order approximation of the curved boundaries is attained by the blending function method [28], enforced directly in the standard square exploiting the same kind of trial functions used for approximating the unknowns. The spectral convergence of the method and the capability of dealing with non-homogeneous and anisotropic media is verified by numerical tests. 


\section{GOVERNING EQUATIONS}

The stationary diffusion of a scalar $u$ (energy, species concentration, ...) is governed by the following conservation equation

$$
\nabla \cdot \mathbf{q}=Q
$$

on a domain $\Omega \subset \mathbb{R}^{d}$ with a piecewise smooth boundary $\partial \Omega$, where $\mathbf{q}$ is the diffusion-flux vector and $Q$ is a source term. A gradient-diffusion model is commonly used to relate the flux vector to the transported scalar, yielding a constitutive equation in the form

$$
\mathbf{q}=-\mathbf{D} \cdot \nabla u
$$

where $\mathbf{D}$ is a symmetric positive-definite diffusivity tensor [29]. The mixed formulation of the scalar diffusion conservation principle stems from equations (1) and (2).The fundamental equation is obtained by substituting (2) into (1). System (1-2) is complemented with Dirichlet boundary conditions (3a) or flux boundary conditions (3b):

$$
\left\{\begin{aligned}
u(\mathbf{x})=g(\mathbf{x}) & \text { for } \mathbf{x} \in \partial \Omega_{g} \\
{[\mathbf{q} \cdot \mathbf{n}](\mathbf{x})=h(\mathbf{x}) } & \text { for } \mathbf{x} \in \partial \Omega_{h}
\end{aligned}\right.
$$

where $\mathbf{n}$ is the outwarding unit normal to the boundary $\partial \Omega_{h}$.

The weak formulation of system (1-2) is obtained through a weighted residual statement, where volume-indicator distributions are chosen as weight functions $[1,23]$. The resulting system of equations for a weight function with support on the single control volume $V \subset \Omega$ reads as

$$
\left\{\begin{array}{l}
\int_{V} \mathbf{D}^{-1} \mathbf{q} \mathrm{d} \mathbf{x}+\int_{\partial V} u^{-} \mathbf{n} \mathrm{d} s=\mathbf{0} \\
\int_{\partial V} \mathbf{q}^{-} \cdot \mathbf{n} \mathrm{d} s=\int_{V} Q \mathrm{~d} \mathbf{x}
\end{array}\right.
$$

Choosing $u, \mathbf{q} \in \mathbb{H}^{1}(V)$ and $\mathbf{D}^{-1} \in \mathbb{L}^{2}(V)$ guarantees the existence of the integrals in (4). In addition, a trace theorem asserts that a function in $\mathbb{H}^{1}(V)$ has a $\mathbb{L}^{2}(\partial V)$ trace $[30$, th. 1.3.1 ch. 1], assuring the existence of the boundary integrals appearing in the weak formulation. It is worth mentioning that $u, \mathbf{q} \in \mathbb{H}^{1}(V)$ and $\mathbf{D}^{-1} \in \mathbb{L}^{2}(V)$ does not imply any continuity constraint between control volumes.

\section{NUMERICAL APPROXIMATION}

\subsection{Domain discretization}

The domain $\Omega \subset \mathbb{R}^{d}$ is subdivided into a triangulation $\mathcal{T}_{h}$ of elements $e$ such that

$$
\bar{\Omega}=\bigcup_{e \in \mathcal{T}_{h}} e
$$


where the subscript $h$ denotes the particular triangulation in which diam $(e) \leq h \forall e \in \mathcal{T}_{h}$ [30, ch. 3.1]. It is assumed that every element $e \in \mathcal{T}_{h}$ can be mapped onto a reference polyhedron $\widehat{e} \subset \mathbb{R}^{d}$ by an invertible transformation (i.e. mapping) $\mathcal{M}_{e}: \widehat{e} \rightarrow e$. In the present work, the chosen reference polyhedron is the standard square $\widehat{e} \equiv[-1,1]^{2} \subset \mathbb{R}^{2}$. In the following, the notations $\mathbf{x} \in \Omega$ and $\boldsymbol{\eta} \in \widehat{e}$ are used to denote the independent variables in the physical and the transformed space, respectively. The hat symbol $\widehat{-}$ denotes geometrical entities in the transformed space $\widehat{e}$, which are mapped onto the physical element $e$ by the mapping $\mathcal{M}_{e}$.

\subsection{High order representation of curved boundaries}

For high order methods it is crucial to describe the curved element's sides with sufficient precision as not to introduce low order errors in the approximation of the unknowns and preserve the method's expected accuracy [31, chap. 3]. In this work, curved elements are dealt with the blending method $[32,33,34]$, which consists in splitting $\mathcal{M}_{e}$ in two contributions, as

$$
\mathcal{M}_{e}=l(\boldsymbol{\eta})+c(\boldsymbol{\eta})
$$

where $l(\boldsymbol{\eta})$ is the mapping for a linear element sharing the same nodes with the actual element, while $c(\boldsymbol{\eta})$ is a correction taking into account the curvilinear sides.

The formulation for the mapping of quadrilateral elements is proposed in [28] and is recalled in section 3.2.1. A different approach is used for mapping triangular elements, as described in detail in section 3.2.2. The approach is described in a general form and the extension to the three-dimensional case is natural.

3.2.1. Quadrilateral curved elements The blending function method applied to a quadrilateral element $e \in \mathcal{T}_{h}$ with only one curved side is sketched in Figure 1. The counter-clockwise convention is used to identify the sides and the vertices of the element. For example, side 1 in the physical space is denoted as $\mathbf{s}_{1}$ and has vertices of coordinates $\mathbf{X}_{A_{1}}=\left(X_{A_{1}}, Y_{A_{1}}\right)$ and $\mathbf{X}_{A_{2}}=\left(X_{A_{2}}, Y_{A_{2}}\right)$ respectively, side 2 is denoted as $\mathbf{s}_{2}$ and so on. Correspondingly in the transformed space $\widehat{e}$, side 1 , denoted as $\widehat{\mathbf{s}}_{1}$, is the linear segment joining vertices $\widehat{A_{1}}=(-1,-1)$ and $\widehat{A_{2}}=(1,-1)$.

The function $l(\boldsymbol{\eta})$ yields the mapping between the linear element and the reference polyhedron and takes the following form

$$
l(\boldsymbol{\eta})=\sum_{i=1}^{4} L_{\widehat{A_{i}}} \mathbf{X}_{A_{i}}
$$

The polynomials $L_{\widehat{A_{i}}}=L_{\widehat{A_{i}}}(\boldsymbol{\eta})$ are linear Lagrange coefficients whose nodes correspond to the vertices $\widehat{A}_{1}, \ldots, \widehat{A}_{4} \in \widehat{e}$. For example, the Lagrange coefficient corresponding to vertex $\widehat{A_{1}}$ reads as

$$
L_{\widehat{A_{1}}}=\frac{1}{4}\left(1-\eta_{1}\right)\left(1-\eta_{2}\right)
$$

Denoting by $\mathbf{s}_{1}\left(\eta_{1}\right)$ the parametric representation of $\mathbf{s}_{1}$ while letting $\mathbf{l}_{\mathbf{s}_{1}}\left(\eta_{1}\right)$ be the restriction of (6) to $\widehat{\mathbf{s}}_{1}$, the term $c(\boldsymbol{\eta})$ of (5) takes the form

$$
c(\boldsymbol{\eta})=\left(\mathbf{s}_{1}-\mathbf{l}_{\mathbf{s}_{1}}\right) F_{1}\left(\eta_{2}\right)
$$




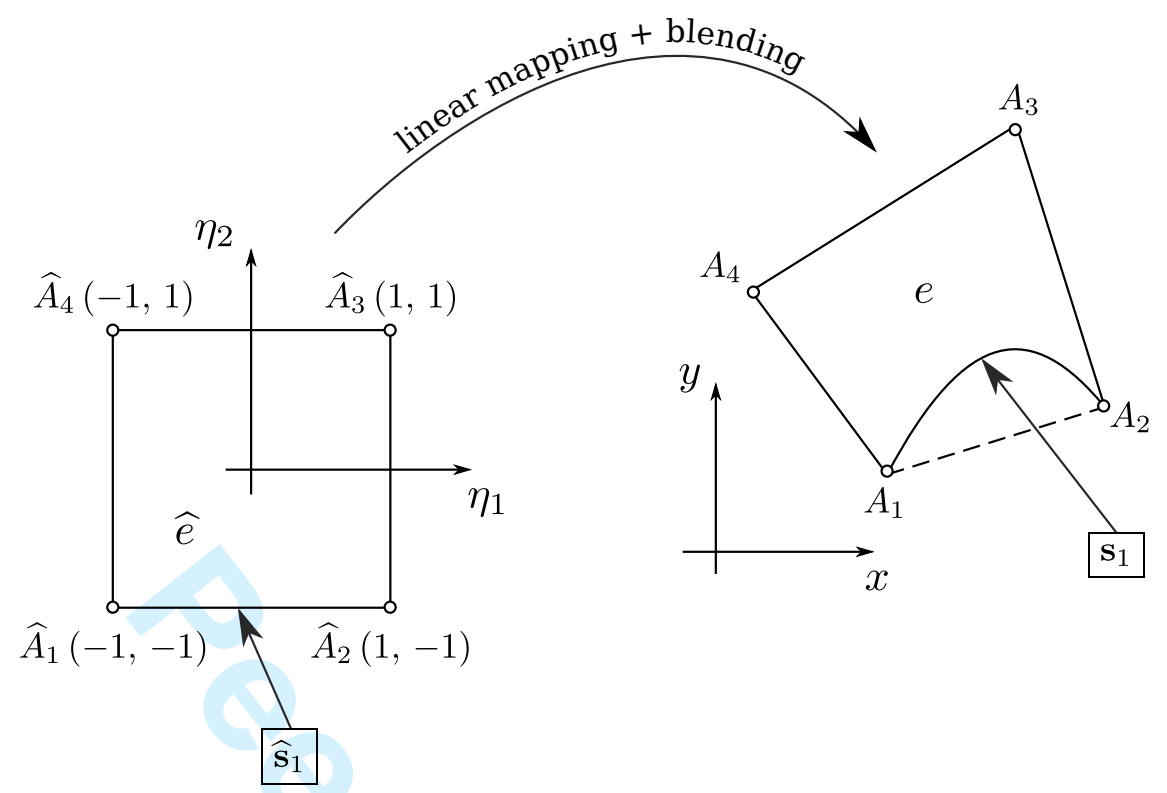

Figure 1. Sketch of the mapping $\mathcal{M}_{e}$ of a quadrilateral element $e \in \mathcal{T}_{h}$ with one curved side, using the blending function method (5).

The term $F_{1}\left(\eta_{2}\right)$ denotes the blending factor associated to side $\mathbf{s}_{1}$, and varies linearly from one, on side $\widehat{\mathbf{s}}_{1}$ (i.e., for $\eta_{2}=-1$ ), to zero, on the opposite side $\widehat{\mathbf{s}}_{3}$ (i.e., for $\eta_{2}=1$ ). The blending factor is introduced with the aim of not affecting the opposite side $\mathbf{s}_{3}$ by the correction $\left(\mathbf{s}_{1}-\mathbf{l}_{\mathbf{s}_{1}}\right)$.

The terms in (8) are computed as:

$$
\begin{aligned}
& \mathbf{s}_{1}=\left.\mathbf{s}_{1}\left(\eta_{1}\right) \approx \sum_{i=1}^{N_{p}} L_{\widehat{p}_{i}}\right|_{\mathbf{s}_{1}} \mathbf{X}_{i}, \mathbf{X}_{i} \in \mathbf{s}_{1} \\
& \mathbf{l}_{\mathbf{s}_{1}}=\left.\sum_{i=1,2} L_{\widehat{A}_{i}}\right|_{\widehat{\mathbf{s}}_{1}} \mathbf{X}_{A_{i}} \\
& F_{1}=\frac{1-\eta_{2}}{2}
\end{aligned}
$$

The curved side definition (9) represents a $\left(N_{p}-1\right)$-th order accurate interpolation of $\mathbf{s}_{1} \cdot N_{p}$ is the number of points that belong to the curved side (including the vertices) and their physical coordinates are denoted by $\mathbf{X}_{i}$. The $\left.L_{\widehat{p}_{i}}\right|_{\widehat{\mathbf{s}}_{1}}$ are $\left(N_{p}-1\right)$-th order Lagrange coefficients restricted to side $\widehat{\mathbf{s}}_{1}$. It is expected that, as not to introduce lower order errors in the geometric approximation, at least $\left(P^{e}+1\right)^{d-1}$ points belonging to the curved side of the physical element $e$ must be provided, with $P^{e}$ being the polynomial order of the trial functions used in the considered element.

In equation (10) for the linear side (dashed line in the physical space in Figure 1) the $\mathbf{X}_{A_{i}}$ are the coordinates of the vertices $A_{1}, A_{2} \in \mathbf{s}_{1}$ in the physical space, and $L_{\widehat{A_{i}}}$ are the respective linear polynomials restricted to $\widehat{\mathbf{s}}_{1} \in \widehat{e}$. Equation (10) reads as

$$
\mathbf{l}_{\mathbf{s}_{1}}=\frac{1-\eta_{1}}{2} \mathbf{X}_{A_{1}}+\frac{1+\eta_{1}}{2} \mathbf{X}_{A_{2}}
$$


In a more general case, all sides of the quadrilateral element can be curvilinear. The expression for the elemental mapping $\mathcal{M}_{e}$ is then obtained by repeating the above procedure to include the contributions of all the sides in the term $c(\boldsymbol{\eta})$ of (5), which in general reads as

$$
c(\boldsymbol{\eta})=\sum_{j=1}^{4}\left(\mathbf{s}_{j}-\mathbf{l}_{\mathbf{s}_{j}}\right) F_{j}
$$

where for $j=1$ equations (9) to (12) are used, while for $j=2,3,4$ we have, respectively:

$$
\begin{aligned}
\mathbf{s}_{2} & =\left.\mathbf{s}_{2}\left(\eta_{2}\right) \approx \sum_{i=1}^{N_{p}} L_{\widehat{p_{i}}}\right|_{\widehat{\mathbf{s}}_{2}} \mathbf{X}_{i}, \mathbf{X}_{i} \in \mathbf{s}_{2} \\
\mathbf{s}_{3} & =\left.\mathbf{s}_{3}\left(\eta_{1}\right) \approx \sum_{i=1}^{N_{p}} L_{\widehat{p_{i}}}\right|_{\widehat{s}_{3}} \mathbf{X}_{i}, \mathbf{X}_{i} \in \mathbf{s}_{3} \\
\mathbf{s}_{4} & =\left.\mathbf{s}_{4}\left(\eta_{2}\right) \approx \sum_{i=1}^{N_{p}} L_{\widehat{p_{i}}}\right|_{\mathrm{s}_{4}} \mathbf{X}_{i}, \mathbf{X}_{i} \in \mathbf{s}_{4} \\
\mathbf{l}_{\mathbf{s}_{2}} & =\left.\sum_{i=2,3} L_{\widehat{A_{i}}}\right|_{\widehat{s}_{2}} \mathbf{X}_{A_{i}}=\frac{1-\eta_{2}}{2} \mathbf{X}_{A_{2}}+\frac{1+\eta_{2}}{2} \mathbf{X}_{A_{3}} \\
\mathbf{l}_{\mathbf{s}_{3}} & =\left.\sum_{i=3,4} L_{\widehat{A_{i}}}\right|_{\widehat{s}_{3}} \mathbf{X}_{A_{i}}=\frac{1+\eta_{1}}{2} \mathbf{X}_{A_{3}}+\frac{1-\eta_{1}}{2} \mathbf{X}_{A_{4}} \\
\mathbf{l}_{\mathbf{s}_{4}} & =\left.\sum_{i=4,1} L_{\widehat{A_{i}}}\right|_{\mathbf{s}_{4}} \mathbf{X}_{A_{i}}=\frac{1+\eta_{2}}{2} \mathbf{X}_{A_{4}}+\frac{1-\eta_{2}}{2} \mathbf{X}_{A_{1}} \\
F_{2}= & \frac{1+\eta_{1}}{2} \quad F_{3}=\frac{1+\eta_{2}}{2} \quad F_{4}=\frac{1-\eta_{1}}{2}
\end{aligned}
$$

3.2.2. Triangular curved elements For triangular curved elements, an elemental mapping $\mathcal{M}_{e}$ associated with the equilateral triangle reference polyhedron is proposed in [28, Chap. 6], exploiting two different sets of coordinates in the form of $\mathcal{M}_{e}=l(\boldsymbol{L})+c\left(\boldsymbol{\xi}_{\text {eq }}, \boldsymbol{L}\right)$, where $\boldsymbol{L}$ and $\boldsymbol{\xi}_{\text {eq }}$ denote two sets of coordinates in two different triangular reference polyhedrons. In particular, $\boldsymbol{L}$ are the so called barycentric or area coordinates, and $\boldsymbol{\xi}_{\text {eq }}$ refer to the equilateral triangle space [28, Chap. 6.3.3].

In the present work, we propose to map triangular elements by the same approach used for quadrilateral elements. The mapping $\mathcal{M}_{e}$ for triangular elements is readily obtained using the same expression (5) by setting $\mathbf{X}_{A_{4}} \equiv \mathbf{X}_{A_{3}}$. The term $l(\boldsymbol{\eta})$ in (5) then becomes

$$
\begin{aligned}
l(\boldsymbol{\eta})=\sum_{i=1}^{4} L_{\widehat{A}_{i}} \mathbf{X}_{A_{i}}= & \frac{1}{4}\left(\eta_{1}-1\right)\left(\eta_{2}-1\right) \mathbf{X}_{A_{1}} \\
& +\frac{1}{4}\left(\eta_{1}+1\right)\left(1-\eta_{2}\right) \mathbf{X}_{A_{2}}+\frac{1}{2}\left(\eta_{2}+1\right) \mathbf{X}_{A_{3}}
\end{aligned}
$$

This approach is equivalent to the Duffy [35] or collapsed coordinates transformation [27] as sketched in Figure 2, yet in the expression of the elemental mapping $\mathcal{M}_{e}$ the auxiliary set of coordinates $\boldsymbol{\xi}=\left(\xi_{1}, \xi_{2}\right)$ of the right triangle space are not used.

Referring to Figure 2, only side $\mathbf{s}_{1}$ is curvilinear. As mentioned, the contribution $c(\boldsymbol{\eta})$ to the mapping of the curvilinear sides in (5) is computed by the same expression used for quadrilateral 


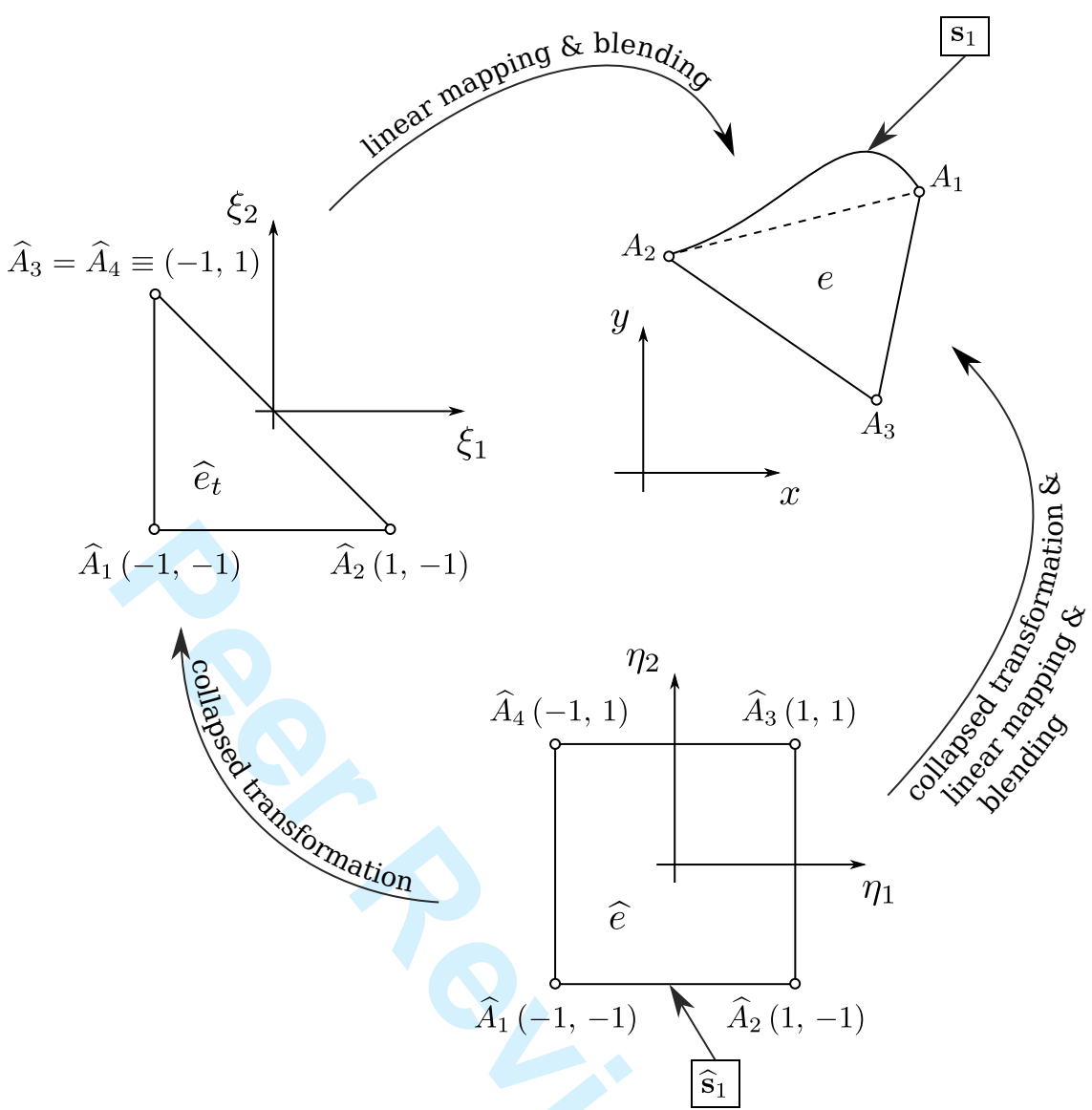

Figure 2. Sketch of the mapping $\mathcal{M}_{e}$ of a triangular element $e \in \mathcal{T}_{h}$ with one curved side, using the blending function method (5). The resulting mapping for triangular elements is the same as the one used for quadrilateral elements.

elements (8), in which the terms are obtained by equations (9) to (12). In the general case when all sides of the triangular element are curvilinear, the computation of the term $c(\boldsymbol{\eta})$ in (5) is complemented by equations (13) to (16), for $j=2,3$.

Some peculiar features of the present approach are worth mentioning:

1. An intermediate collapsed coordinate mapping between the standard square and the standard triangle [31, ch. 3] is not used, resulting in a simpler and more general formulation;

2. The same kind of interpolatory functions $L$ can be used both as shape functions for the approximation of the unknowns and for the interpolation of the curved sides;

3. In the formulation proposed by [28] for triangular elements, the blending factors involve higher order polynomials and lead to a non-linear variation of the internal isolines of constant $\eta_{1}, \eta_{2}$; whereas, by applying the blending method directly in the transformed reference space $\widehat{e}=[-1,1]^{d}$, the resulting blending factors $F_{j}$ in equation (13) are always linear polynomials and the isolines vary linearly in the interior of the element (see Figure 4). 


\subsection{Finite dimensional subspace and basis functions}

The unknowns $u(\mathbf{x})$ and $\mathbf{q}(\mathbf{x})$ are approximated by element-based polynomial expansions [13]. Let $\mathbb{Q}^{k, d}$ be the finite dimensional space whose elements $v_{h} \in \mathbb{Q}^{k, d}$ are polynomial functions from $\mathbb{R}^{d}$ to $\mathbb{R}$ of degree less than or equal to $k$ in each variable $\eta_{1}, \ldots, \eta_{d}, k \geq 0$ [30, Chap. 3.2]. Considering the restriction to the reference polyhedron $\widehat{e}=[-1,1]^{d}$ we define the space

$$
\mathbb{Q}_{\widehat{e}}^{k, d}:=\left\{\left.v_{h}\right|_{e} \text { s.t. } v_{h} \in \mathbb{Q}^{k, d}\right\}
$$

The approximation $u_{h}$ to $u$ is chosen in the finite-dimensional subspace $X_{h}^{k, d}$ of $\mathbb{H}^{1}(\Omega)$, with $\Omega \subset \mathbb{R}^{d}$, defined as

$$
X_{h}^{k, d}:=\left\{w_{h}: \Omega \rightarrow \mathbb{R} \text { s.t. }\left.w_{h}\right|_{e} \equiv v_{h} \circ \mathcal{M}_{e}^{-1} \forall e \in \mathcal{T}_{h}, v_{h} \in \mathbb{Q}_{e}^{k, d}\right\}
$$

while $\mathbf{q}_{h}$ is chosen in $\left(X_{h}^{k, d}\right)^{d}$.

An interpolatory basis for the space $X_{h}^{k, d}$ is constructed as follows. Letting $i \equiv(e, l), l=1, \ldots$, $\left(P^{e}+1\right)^{d}$, in the present work the basis functions of $X_{h}^{k, d}$ are defined as:

$$
\phi_{i}(\mathbf{x})= \begin{cases}L_{e, l} \circ \mathcal{M}_{e}^{-1}(\mathbf{x}), & \mathbf{x} \in \bar{e} \\ 0, & \mathbf{x} \in \bar{\Omega} \backslash \bar{e}\end{cases}
$$

Accordingly, we have

$$
\phi_{i}\left(\mathbf{X}_{j}\right)=\delta_{i j}, \quad i, j=1, \ldots, N_{h}
$$

where $\mathbf{X}_{j}$ denotes an interpolation node, $j, \ldots, N_{h}$, with $N_{h}$ the total number of interpolation nodes in the mesh.

In principle, the interpolation nodes may be located everywhere inside an element. Nevertheless, Gauss-Legendre or Gauss-Lobatto-Legendre quadrature nodes result in general in well-conditioned systems of equations $[15,31,1]$ and are used in the present study (see Figure 3).

\subsection{Inter-element coupling and boundary conditions}

On the element's boundary, numerical fluxes $\widetilde{u}_{h}$ and $\widetilde{\mathbf{q}}_{h}$ have to be defined in such a way that the resulting discontinuous method is consisted and convergent $[12,13]$. The local discontinuous Galerkin (LDG) formulation is used [26]:

$$
\begin{aligned}
\widetilde{u}_{h} & =\left\{u_{h}\right\} \\
\widetilde{\mathbf{q}}_{h} & =\left\{\mathbf{q}_{h}\right\}+C_{11} \llbracket u_{h} \rrbracket
\end{aligned}
$$

where $C_{11}>0$. The operators $\{\cdot\}$ and $\llbracket \cdot \rrbracket$ denote the inter-element average and jump, respectively:

$$
\begin{array}{ll}
\left\{u_{h}\right\} \equiv \frac{1}{2}\left(u_{h}{ }^{-}+u_{h}{ }^{+}\right) & \left\{\mathbf{q}_{h}\right\} \equiv \frac{1}{2}\left(\mathbf{q}_{h}{ }^{-}+\mathbf{q}_{h}{ }^{+}\right) \\
\llbracket u_{h} \rrbracket \equiv\left(u_{h}{ }^{-} \mathbf{n}^{-}+u_{h}{ }^{+} \mathbf{n}^{+}\right) & \llbracket \mathbf{q}_{h} \rrbracket \equiv\left(\mathbf{q}_{h}{ }^{-} \cdot \mathbf{n}^{-}+\mathbf{q}_{h}{ }^{+} \cdot \mathbf{n}^{+}\right)
\end{array}
$$


where the "-" and "+" signs denote the one-sided limits of the function when approaching the element's boundary $\partial e$, and $\mathbf{n}^{-}$and $\mathbf{n}^{+}$denote the respective outwarding normals unit vectors. On the domain's boundary $\partial \Omega$, the following distinct formulations apply:

Dirichlet boundary $\partial \Omega_{D} \quad$ According to [12]:

$$
\begin{aligned}
\widetilde{u}_{h} & =g(\mathbf{x}) \\
\widetilde{\mathbf{q}}_{h} & =\mathbf{q}_{h}-C_{11} \llbracket u_{h} \rrbracket
\end{aligned}
$$

where the change of sign in (24b) compared to (22b) results in [12].

Flux boundary $\partial \Omega_{N}$ According to [13]:

$$
\begin{aligned}
\widetilde{T}_{h} & =T_{h}(\partial \Omega) \\
\widetilde{\mathbf{q}}_{h} \cdot \mathbf{n} & =f(\mathbf{x})
\end{aligned}
$$

\subsection{Dual mesh: partition into control volumes}

Each element $e$ is subdivided into a partition of $\left(P^{e}+1\right)^{d}$ control volumes $V$. Differently from continuous CVFEM methods, each control volume is entirely contained in a single element. The resulting ensemble of control volumes $V$ can be seen as a dual mesh with respect the primary triangulation $\mathcal{T}_{h}$.

In principle any partition into control volumes can be used. However, in the proposed formulation the control volume sides lay on $-1,+1$ and on $P^{e}-1$-th order Gauss-Legendre quadrature nodes of the one-dimensional reference element $\widehat{e} \equiv[-1,1]$. The extension to two- or three-dimensions is easily obtained by tensor-product of the one-dimensional element in the transformed space. Figure 3 shows the partition of the canonical element $\widehat{e} \equiv[-1,1]^{2}$ into control volumes for different polynomial orders of accuracy $P^{e}$.

\section{ANALYSIS OF THE MATRIX CONDITION NUMBER}

In testing the convergence of high order methods, the accumulation of round-off errors can be large compared to the discretization error especially for higher orders of accuracy. The tendency of the method to provide round off errors in the solution can be estimated through the analysis of the condition number of the matrix resulting from the discretization process. The geometric properties of the mesh affect the condition number, and it is recommended the use of elements of nearly equal size and having almost the same lengths in each dimension [36]. In particular, when dealing with non-stationary problems, severe limitations are posed by the conditioning of the mass matrix. In the present analysis the condition number of the elemental mass matrix $\mathbf{M}^{e}$ is computed and the influence of the elemental mapping (section 3.2) is investigated by considering different geometrical configurations. The contribution of the element $e$ to the mass matrix for the DCVFEM is calculated as

$$
\mathbf{M}_{i j}^{e}=\int_{V_{i}} \phi_{j}(\mathbf{x}) \mathrm{d} \mathbf{x}=\int_{\widehat{V}_{i}} L_{j}(\boldsymbol{\eta})\left|\mathbf{J}_{\mathbf{x}}\right| \mathrm{d} \boldsymbol{\eta}
$$




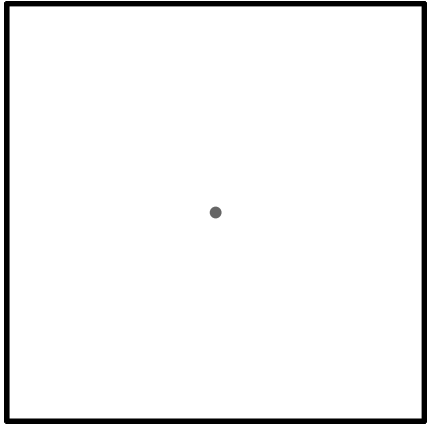

(a) $P^{e}=0$

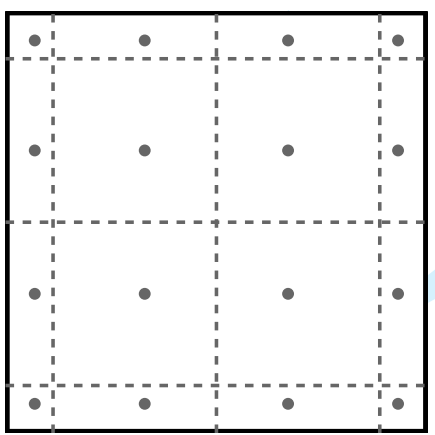

(d) $P^{e}=3$

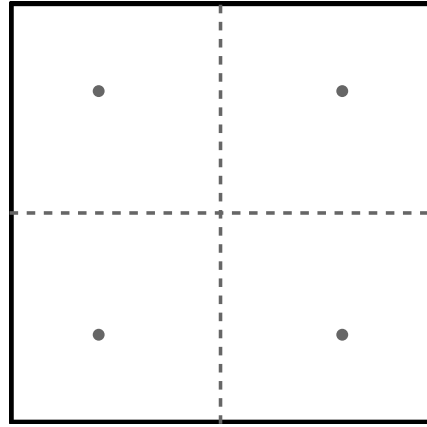

(b) $P^{e}=1$

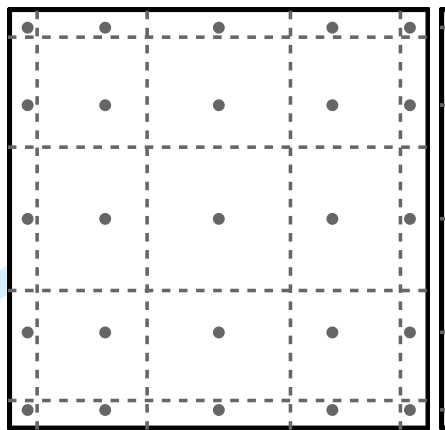

(e) $P^{e}=4$

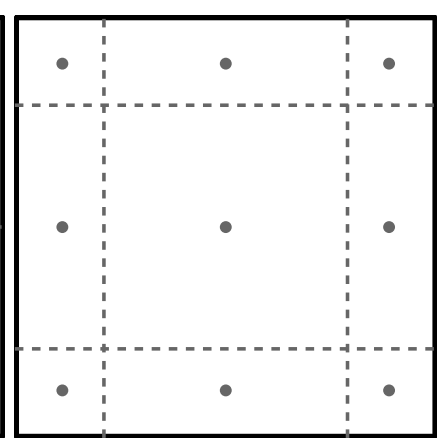

(c) $P^{e}=2$

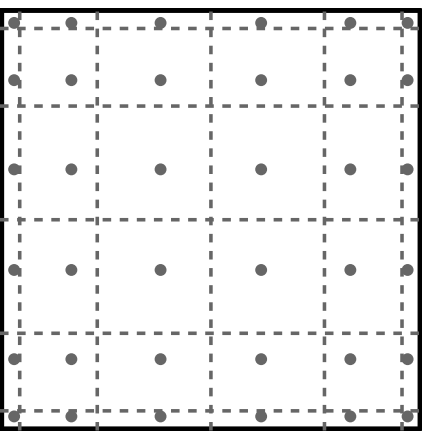

(f) $P^{e}=5$

Figure 3. Partition of the canonical element $\widehat{e} \equiv[-1,1]^{2}$ into $\left(P^{e}+1\right)^{2}$ control volumes (dashed lines) for different polynomial orders $P^{e}$. The corresponding interpolation nodes (dots) are sketched.

where $\mathrm{V}_{i}$ and $\widehat{V}_{i}$ denote the i-th control volume in the physical and transformed spaces, respectively. The Lagrange coefficient $\phi_{j}$ in the physical space is associated to its corresponding Lagrange coefficient in the transformed space by the elemental mapping as $\phi_{j}=L_{j} \circ \mathcal{M}_{e}^{-1}$ (see section 3.3). The Jacobian of the elemental mapping is denoted by $\mathbf{J}_{\mathbf{x}}=\mathbf{J}_{\mathbf{x}}(\boldsymbol{\eta})=\partial x_{k} / \partial \eta_{l}$, with $k, l=1, \ldots, d$, the operator $|\cdot|$ denotes the determinant. For the two-dimensional case, let $j \equiv\left(j_{1}, j_{2}\right), L_{j}$ is obtained by tensorial product of one-dimensional interpolants:

$$
\mathbf{M}_{i j}^{e}=\int_{\widehat{V}_{i}} L_{j_{1}}\left(\eta_{1}\right) L_{j_{2}}\left(\eta_{2}\right)\left|\mathbf{J}_{\mathbf{x}}\right| \mathrm{d} \boldsymbol{\eta}, \quad 1 \leq j_{1}, j_{2} \leq P^{e}+1
$$

Quadrilateral and triangular elements in geometrically highly distorted configurations are compared against the square and the equilateral triangular element, respectively. The selected elements are illustrated in Figure 4. The control volume boundaries are plotted to envision the effect of the mapping in the interior of the element. For the selected highly distorted configurations of the elements, it has been verified that the Jacobian determinant $\left|\mathbf{J}_{\mathbf{x}}\right|$ never changes sign and is always positive, thus the mapping $\mathcal{M}_{e}$ is invertible.

The condition number of the elemental mass matrix in (27) is calculated as

$$
\kappa\left(\mathbf{M}_{i j}^{e}\right)=\left\|\mathbf{M}_{i j}^{e}\right\|_{p}\left\|\left(\mathbf{M}_{i j}^{e}\right)^{-1}\right\|_{p}
$$




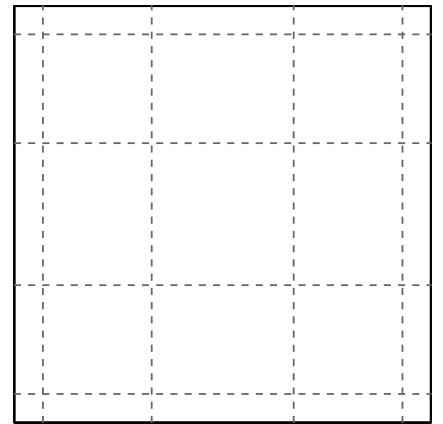

(a)

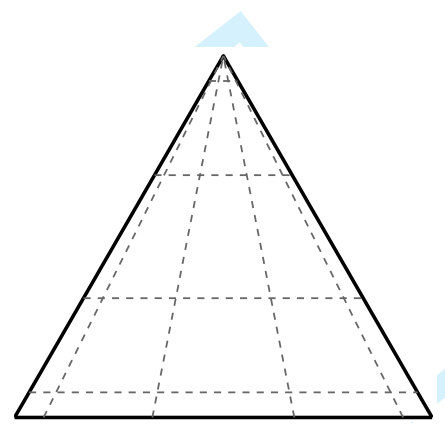

(d)

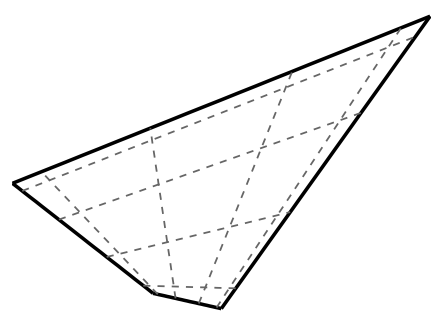

(b)

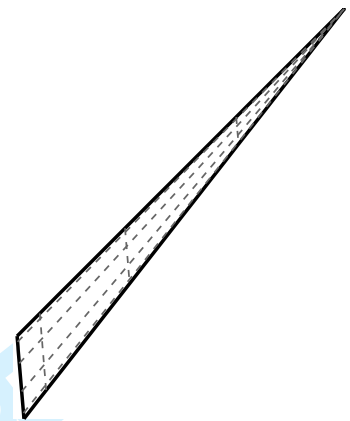

(e)

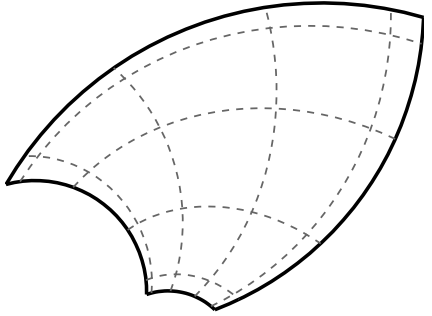

(c)

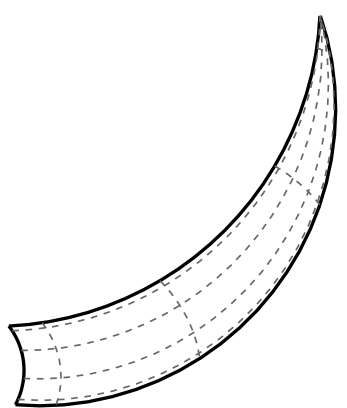

(f)

Figure 4. Regular, distorted and curved elements: (a-c) quadrilateral element; (d-f) triangular element. The boundaries of the control volumes for $P^{e}=4$ are plotted for reference.

where the $\|\cdot\|_{p}$ denotes the matrix norm with $p=2$ and $p=\infty$. A low condition number indicates a well-conditioned matrix which can be readily inverted and vice versa. Typically, in spectral methods the condition number grows exponentially with the polynomial order [31]. Figure 5 shows the computed mass matrix condition number in 2- and $\infty$-norm for the quadrilateral and triangular element, with polynomial order up to $P^{e}=10$. The following remarks can be made:

- The quadrilateral elements (Figure 5a) exhibit the best conditioning. The condition number grows with the polynomial order at a rate roughly $k \propto\left(P^{e}\right)^{2}$ and $k \propto\left(P^{e}\right)^{3}$ for the regular and highly distorted/curved configuration, respectively;

- For the triangular element (Figure 5b), the condition number exhibits a growth rate of roughly $k \propto\left(P^{e}\right)^{4}$ regardless the regular or distorted/curved configuration. However, for polynomial orders up to $P^{e}=4$ the condition number is comparable to the quadrilateral case;

- Even in the most unfavorable configuration of distorted/curved element, the condition number's growth rate is bounded by $k \propto\left(P^{e}\right)^{4}$.

\section{NUMERICAL EXPERIMENTS}

\subsection{Concentric circular disks: convergence study}

The case of homogeneous diffusion inside a section of a hollow circular cylinder is considered. Due to radial symmetry, only one quarter of the domain is solved, as sketched in Figure 6a. The problem 


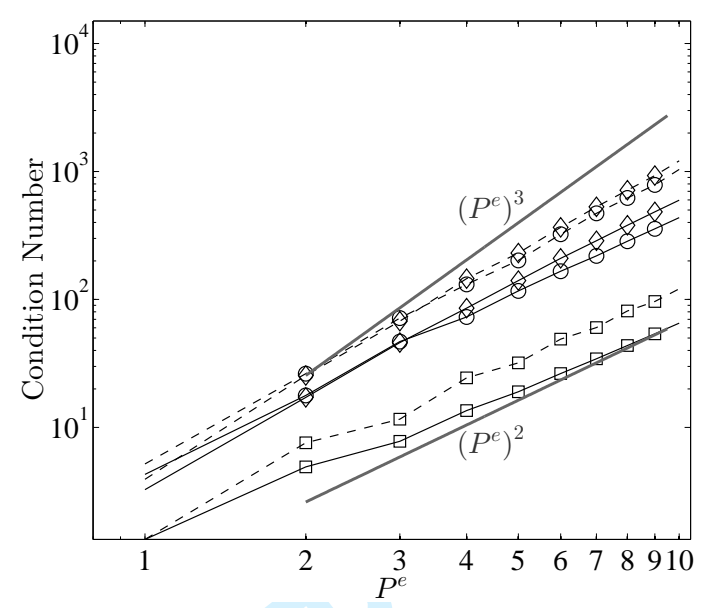

(a)

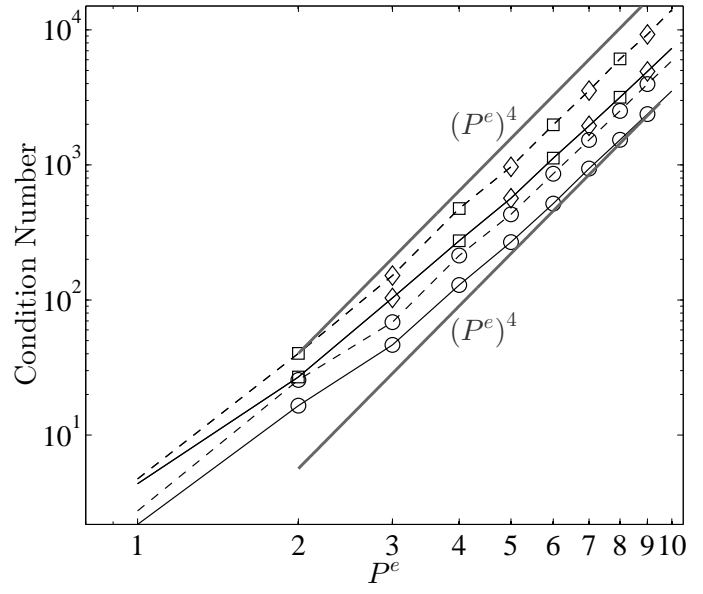

(b)

Figure 5. Mass matrix condition number for the (a) quadrilateral and (b) triangular element. Symbols (see Figure 4): $\square$ regular; $\diamond$ distorted; $\bigcirc$ distorted and curved. Lines: ( - ) matrix 2-norm; (- - -) matrix $\infty$-norm; (—) reference.

is defined by system (1-2) complemented with the following Dirichlet and flux boundary conditions

$$
\left\{\begin{array}{l}
u\left(\mathbf{x} \in \Gamma_{D 1}\right)=u_{1} \\
u\left(\mathbf{x} \in \Gamma_{D 2}\right)=u_{2} \\
{[\mathbf{q} \cdot \mathbf{n}]\left(\mathbf{x} \in \Gamma_{N 1}, \Gamma_{N 2}\right)=0}
\end{array}\right.
$$

with zero source term $Q=0$ and uniform thermal diffusion coefficient $\mathbf{D}=\mathbf{I}$. The analytic solutions for the scalar and vector quantities are given, respectively, as

$$
\begin{aligned}
u(\mathbf{x}) & =\frac{u_{2}-u_{1}}{\ln \left(R_{2} / R_{1}\right)}\left(\ln \left(\|\mathbf{x}\|_{2}\right)-\ln \left(R_{1}\right)\right)+u_{1} \\
\mathbf{q}(\mathbf{x}) & =\frac{u_{1}-u_{2}}{\|\mathbf{x}\|_{2} \ln \left(R_{2} / R_{1}\right)} \frac{\mathbf{x}}{\|\mathbf{x}\|_{2}}
\end{aligned}
$$

This test case is aimed at verifying the exponential convergence of the proposed DCVFEM in presence of curved boundaries. The optimal expected convergence is of $h^{P^{e}+1}$ for the scalar $u$ and $h^{P^{e}}$ for the vector quantity $\mathbf{q}$ as for the DGFEM [37], with $h$ denoting the mesh size. Since sides $\Gamma_{D 1}$ and $\Gamma_{D 2}$ are circular arcs, thus not polynomial expressions, the geometry cannot be described exactly by the chosen geometrical shape functions (Lagrange coefficients). It is therefore expected that the optimal convergence can be achieved only by employing an equal (or superior) order of accuracy in approximating the curved sides of the domain as to avoid "polluting" the solution by lower-order errors.

The $L_{2}$ norm is used to estimate the error

$$
e_{L_{2}}=\frac{\left\|u-u_{h}\right\|_{L_{2}}}{\|u\|_{L_{2}}}=\frac{\left[\int_{\Omega}\left(u(\mathbf{x})-u_{h}(\mathbf{x})\right)^{2} \mathrm{~d} \mathbf{x}\right]^{1 / 2}}{\left[\int_{\Omega}(u(\mathbf{x}))^{2} \mathrm{~d} \mathbf{x}\right]^{1 / 2}}
$$




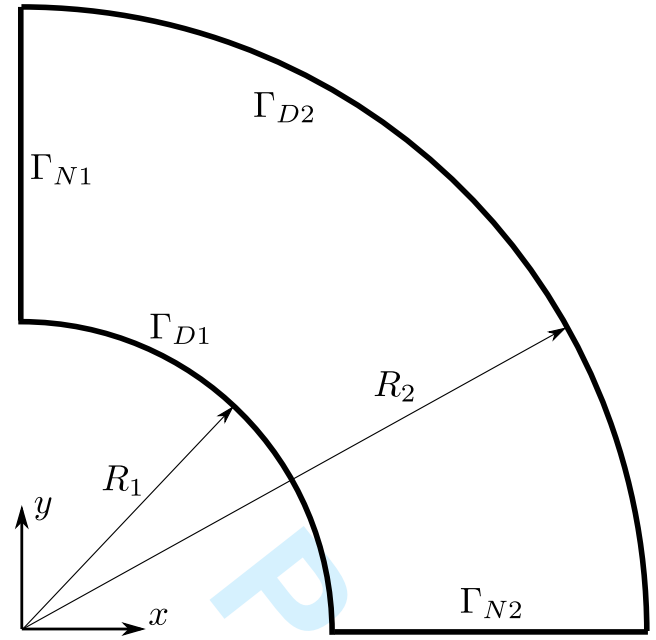

(a) Sketch of the domain

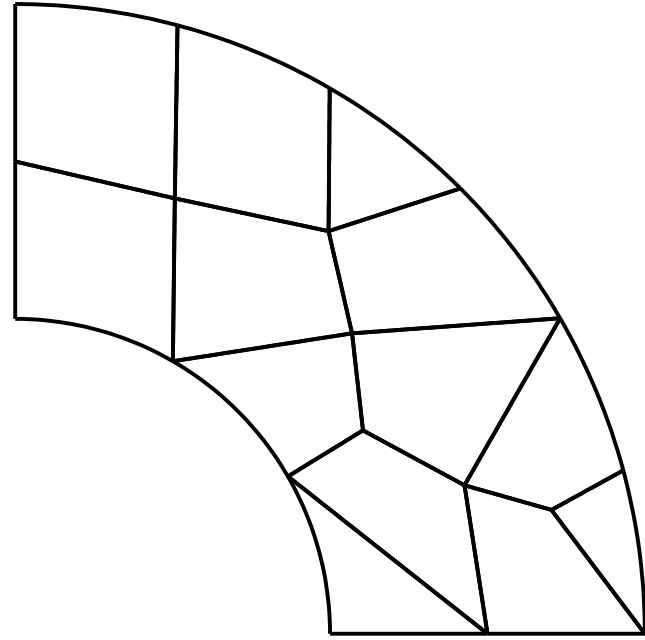

(b) Example of hybrid mesh with curved elements

Figure 6. Homogeneous diffusion problem: system (1-2) coupled with (29) on the hollow circular cylinder section. Due to radial symmetry, only one quarter of the domain is solved.

System (1-2) coupled with boundary conditions (29) is solved by the DCVFEM with polynomial orders ranging from $P^{e}=1$ to $P^{e}=6$ and using both hybrid meshes (mixed quadrilateral and triangular elements, as shown in Figure 6b) and triangular meshes. The $L_{2}$ norm errors (31) are plotted in Figures 7 and 8 for different polynomial orders $P^{e}$ and mesh sizes $h$. In the presented results, the same order of accuracy $P^{e}$ is employed for both the geometry and the unknowns. The lines of optimal convergence $\left(h^{P^{e}+1}\right.$ for the scalar $T$ and $h^{P^{e}}$ for the vector $\left.\mathbf{q}\right)$ and super-convergence ( $h^{P^{e}+2}$ for the scalar $T$ and $h^{P^{e+1}}$ for the vector $\mathbf{q}$ ) are plotted for reference with solid thin line and dashed line, respectively. The following conclusions can be drawn:

- The expected optimal order of convergence is achieved for both the scalar and the vector quantity using a hybrid mesh (Figure 7);

- When using a triangular mesh, super-convergence is achieved for both the scalar and the vector quantity for certain polynomial orders $P^{e}>1$;

- In Figures 7a and 8a, the error decay rate is significantly reduced for $P^{e}=5,6$ and fine meshes. This effect is typically observed in spectral methods when higher polynomial orders are employed [31], since the worse conditioning of the system (see Section 4) is associated with an augmented sensitivity to round-off errors.

For the present numerical experiment, it has been verified that the convergence rate of the solution is determined by the lowest order of accuracy among the approximation of the geometry and the approximation of the unknowns. For instance, if the curved geometry is described with an order of accuracy $P^{g}<P^{e}$, then the convergence of the solution is of $O\left(h^{P^{g}+1}\right)$ for the scalar and $O\left(h^{P^{g}}\right)$ for the vector quantity, respectively. The results are not shown for brevity. 


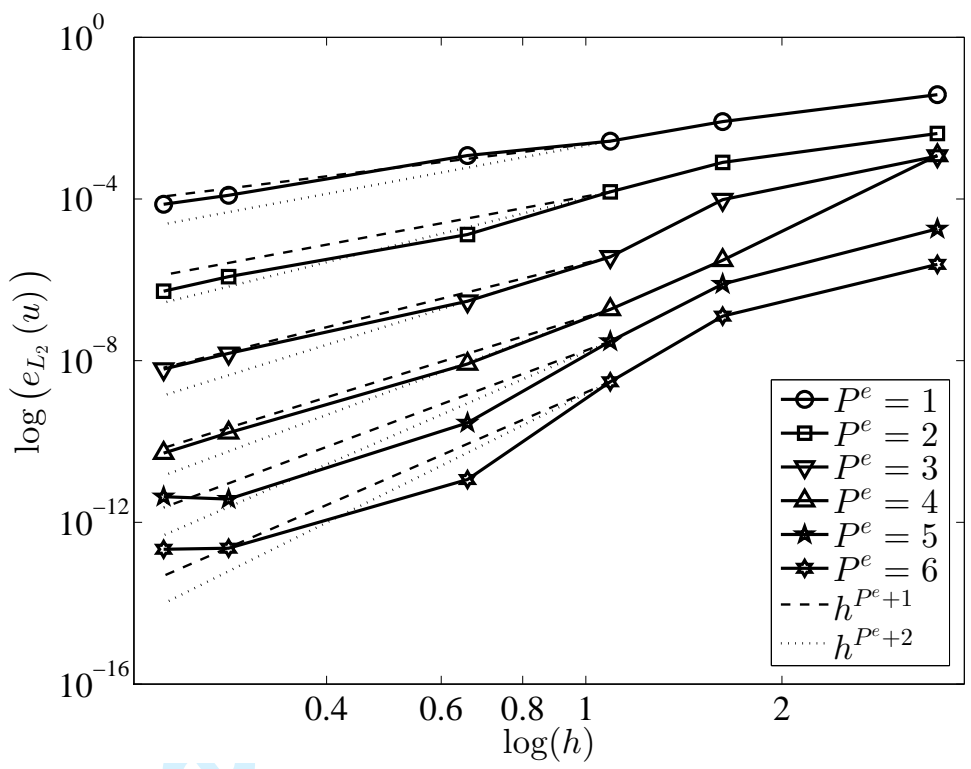

(a) Hybrid mesh: $h$-convergence of the scalar $u$

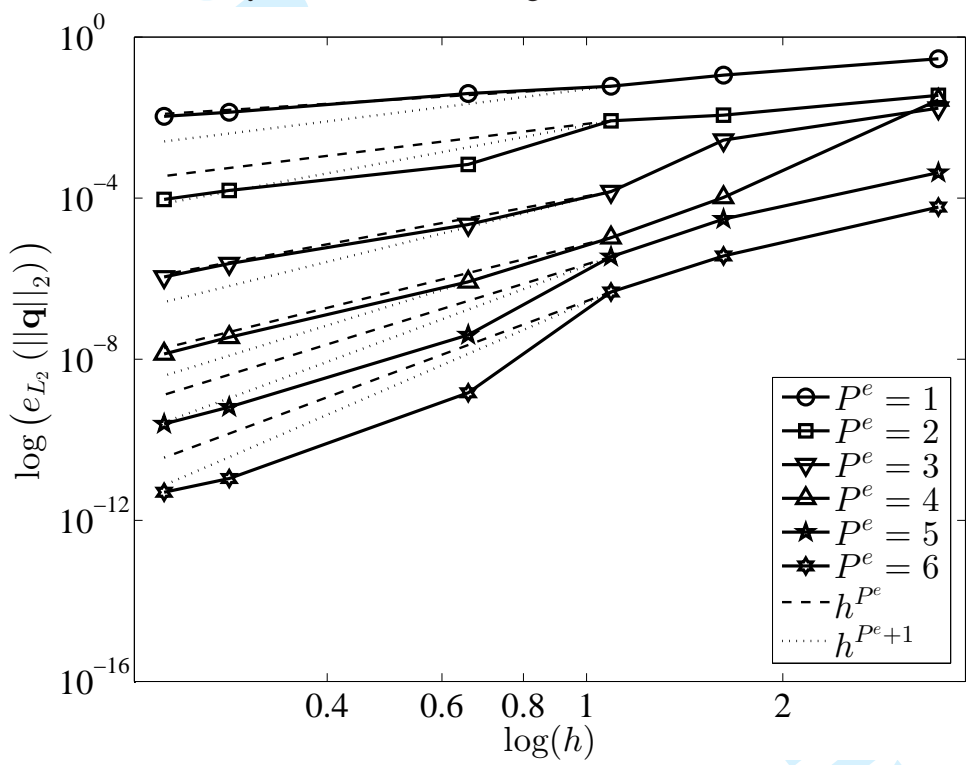

(b) Hybrid mesh: $h$-convergence of the flux $\mathbf{q}$

Figure 7. $L_{2}$ norm errors (31) for the solution of system (1-2) coupled with (29) using a hybrid mesh. $h$ convergence of (a) the scalar $u$ and (b) the vector q. Lines of optimal convergence rate (dashed line) and super-convergence rate (dotted line) are plotted for reference. 


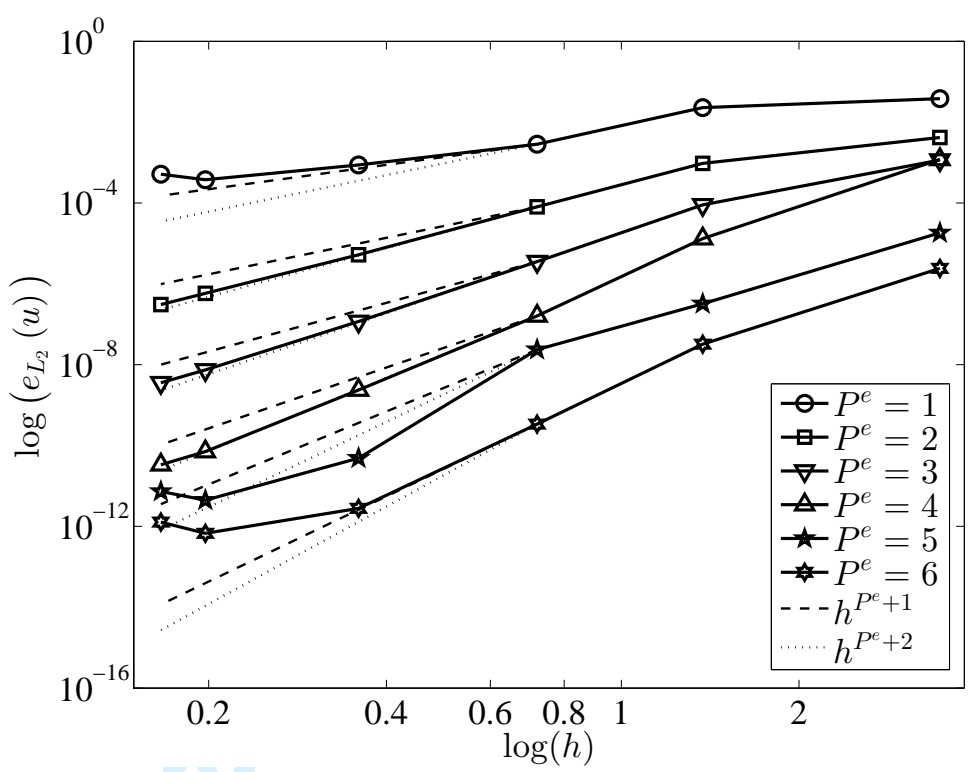

(a) Triangular mesh: $h$-convergence of the scalar $u$

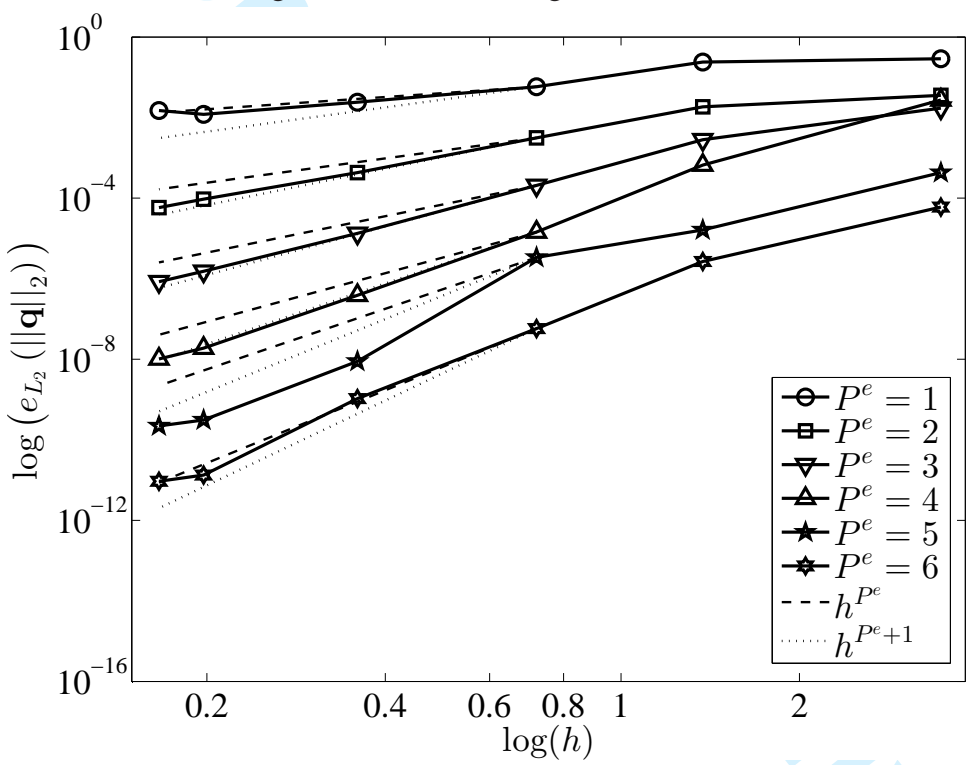

(b) Triangular mesh: $h$-convergence of the flux $\mathbf{q}$

Figure 8. $L_{2}$ norm errors (31) for the solution of problem (1-2) coupled with (29) using a triangular mesh. $h$-convergence of (a) the scalar $u$ and (b) the vector q. Lines of optimal convergence rate (dashed line) and super-convergence rate (dotted line) are plotted for reference.

\subsection{Non-homogeneous diffusion}

The problem of diffusion in an inhomogeneous media considered by [38] is defined by system (1-2) coupled with zero Dirichlet boundary conditions and a source term as

$$
\left\{\begin{array}{l}
u\left(\mathbf{x} \in \Gamma_{D}\right)=0 \\
Q(\mathbf{x})=-2\left(\left(x^{2}-2 x\right)+\left(y^{2}-2 y\right)\right) \\
\mathbf{D}\left(\mathbf{x} \in \Omega \backslash \Omega_{s}\right)=\mathbf{I} \\
\mathbf{D}\left(\mathbf{x} \in \Omega_{s}\right)=10^{-2} \cdot \mathbf{I}
\end{array}\right.
$$




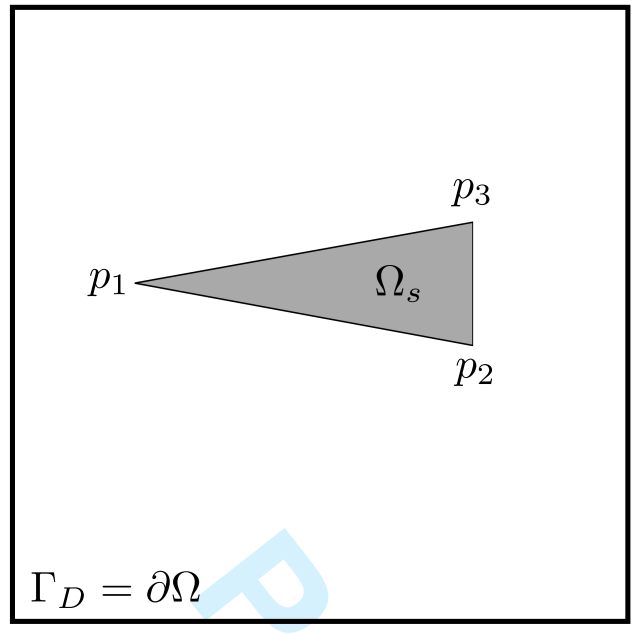

(a) Sketch of the domain

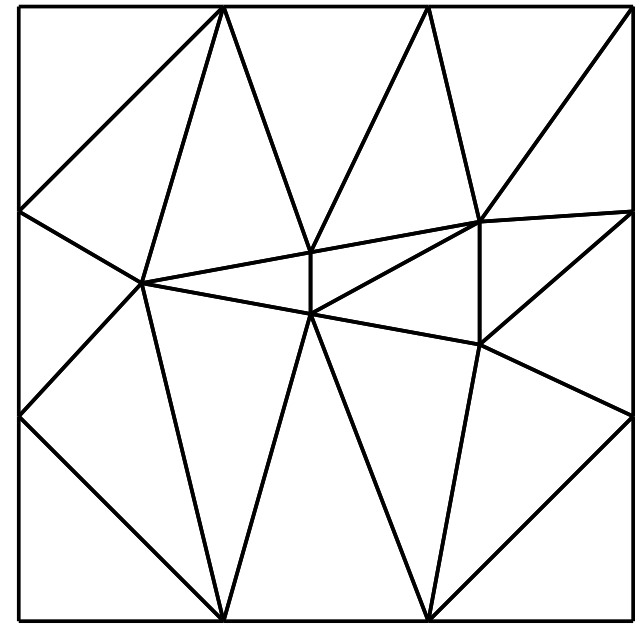

(b) Coarse triangular mesh of 20 elements

Figure 9. Non-homogeneous diffusion problem: system (1-2) coupled with (32).

where $\Omega$ is the square $(0,2) \times(0,2)$ and the triangular inclusion $\Omega_{s}$ is specified by the three vertices $p_{1}=(0.4,1.1), p_{2}=(1.5,0.9), p_{3}=(1.5,1.3)$, as sketched in Figure 9a.

The present test case is motivated by the difficulty in capturing the large gradient in the solution $u$ caused by the discontinuous diffusivity $\mathbf{D}$, which varies abruptly in a narrow portion of the domain. The proposed DCVFEM is used to solve the problem on two different meshes of 20 and 164 triangular elements. The coarse mesh is shown in Figure 9b. Varying polynomial orders are used. The solution for the scalar quantity $u$ is illustrated in Figure 10. Figures 10a and 10b show the solution on a coarse and fine triangular mesh with linear elements $P^{e}=1$, respectively. The solution on the coarse mesh and with elements of order $P^{e}=2$ and $P^{e}=3$ is shown in Figure 10c and 10d, respectively. The following conclusions can be drawn:

- The large gradient in the solution is not captured by a second-order accurate approximation $P^{e}=1$ on the coarser mesh, as can be seen from Figure 10a, and poorly captured when employing a fine mesh of 164 elements, as illustrated in Figure 10b. This phenomenon may be partially explained by the fact that linear polynomials $P^{e}=1$ are used for the approximation of a quadratic source term (see the expression of $Q$ in (32)). On the other hand, quadratic and cubic elements significantly improve the solution although a very coarse mesh of 20 elements is used, as shown in Figure 10c and 10d, respectively. The number of degrees of freedom (d.o.f.) per element is given by $N_{\text {d.o.f }}^{e}=N_{u} \cdot\left(P^{e}+1\right)^{d}$, where $N_{u}=3$ denotes the number of unknowns (in this case one scalar quantity $u$ and two components of the vector quantity q). Accordingly, the total number of d.o.f. involved in the four cases is $N_{\text {d.o.f }}=240,1968,540,960$. A fourth-order accurate simulation $P^{e}=3$ (see Figure 10d) achieves a dramatically sharp and clear capturing of the discontinuity in comparison to the second-order accurate case $P^{e}=1$ on a fine mesh (see Figure 10b), by involving only roughly half the total number of d.o.f. It is apparent that the straightforward p-refinement capability [12] of the proposed DCVFEM may be of crucial importance in applications involving large gradients and discontinuities; 


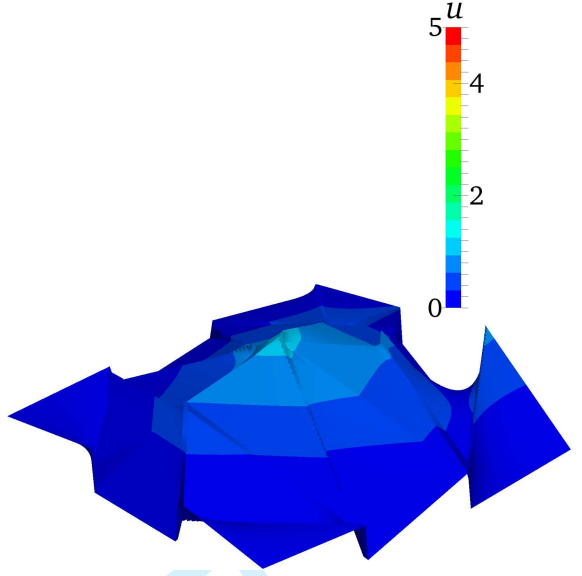

(a) $P^{e}=1$ on a coarse mesh of 20 elements, $N_{\text {d.o.f }}=240$

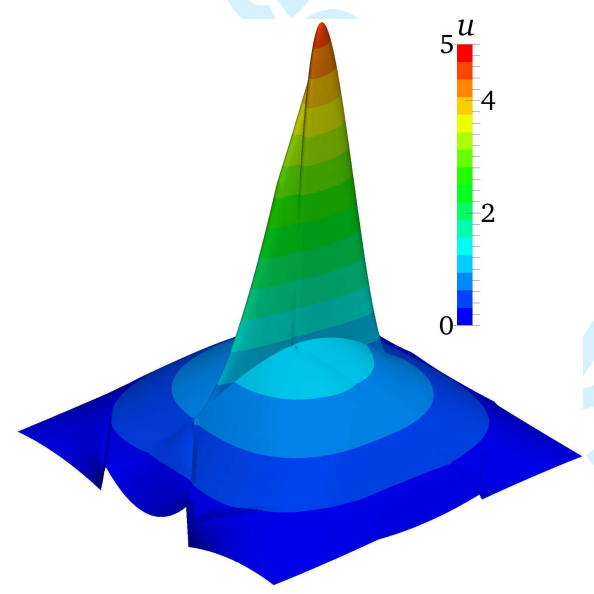

(c) $P^{e}=2$ on a coarse mesh of 20 elements,

$$
N_{\text {d.o.f }}=540
$$

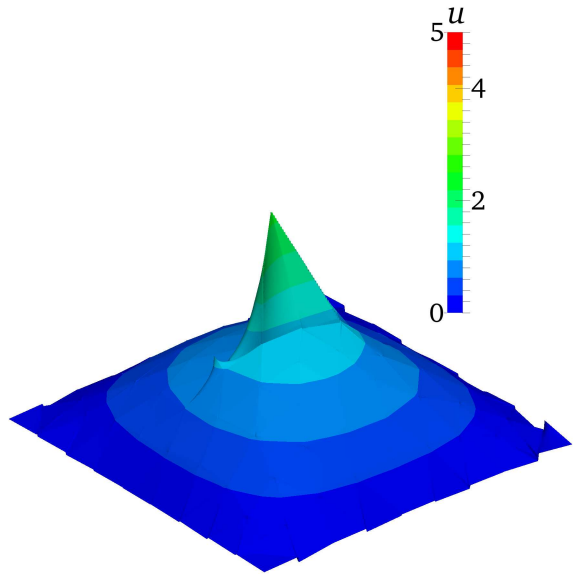

(b) $P^{e}=1$ on a fine mesh of 164 elements, $N_{\text {d.o.f }}=1968$

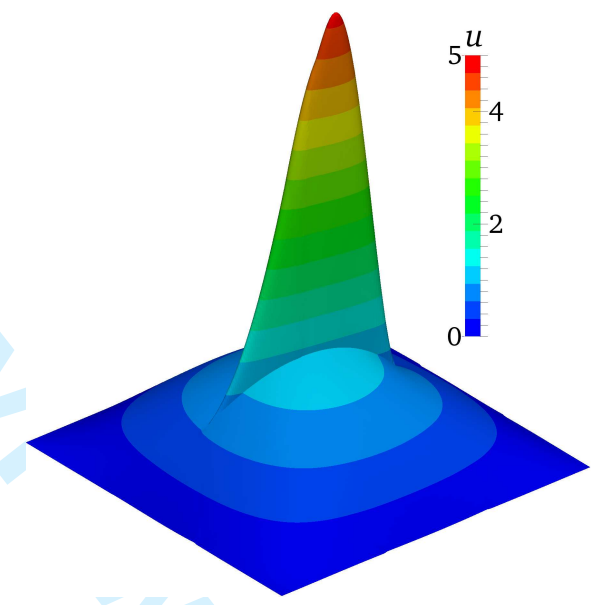

(d) $P^{e}=3$ on a coarse mesh of 20 elements, $N_{\text {d.o.f }}=960$

Figure 10. Solution to problem (1-2) coupled with (32): distribution of the scalar $u$.

- As the resolution is increased, either by $h$ - or $p$-refinement, the proposed DCVFEM solution is free from unphysical oscillations around the discontinuity in the diffusivity $\mathbf{D}$.

\subsection{Anisotropic diffusion}

Diffusion in an anisotropic and non-homogeneous media is considered [38, 39]. System (1-2) is solved on a square domain $\Omega=(0,1) \times(0,1)$, coupled with Dirichlet boundary conditions 
described by the following continuous piecewise linear function on $\partial \Omega$

$$
T\left(\mathbf{x} \in \Gamma_{D}\right)=\left\{\begin{array}{llll}
0 & \text { for } & \mathbf{x} \in(0,0.2) \times\{0\} \cup\{0\} \times(0,0.2) \\
1 & \text { for } & \mathbf{x} \in(0.8,1) \times\{1\} \cup\{1\} \times(0.8,1) \\
0.5 & \text { for } & \mathbf{x} \in(0.3,1) \times\{0\} \cup\{0\} \times(0.3,1) \\
0.5 & \text { for } & \mathbf{x} \in(0,0.7) \times\{1\} \cup\{1\} \times(0,0.7) \\
1 . i . & \text { for } & \mathbf{x} \in(0.2,0.3) \times\{0\} \cup\{0\} \times(0.2,0.3) \\
1 . i . & \text { for } & \mathbf{x} \in(0.7,0.8) \times\{1\} \cup\{1\} \times(0.7,0.8)
\end{array}\right.
$$

where 1.i. denotes the linear interpolation between the values of the adjacent piecewise constant segments. The domain contains a square inclusion $\Omega_{s}=(0.3,0.6) \times(0.3,0.6)$, denoted by the shaded area in Figure 11a. A coarse hybrid mesh of 27 elements is used, as shown in Figure 11b.

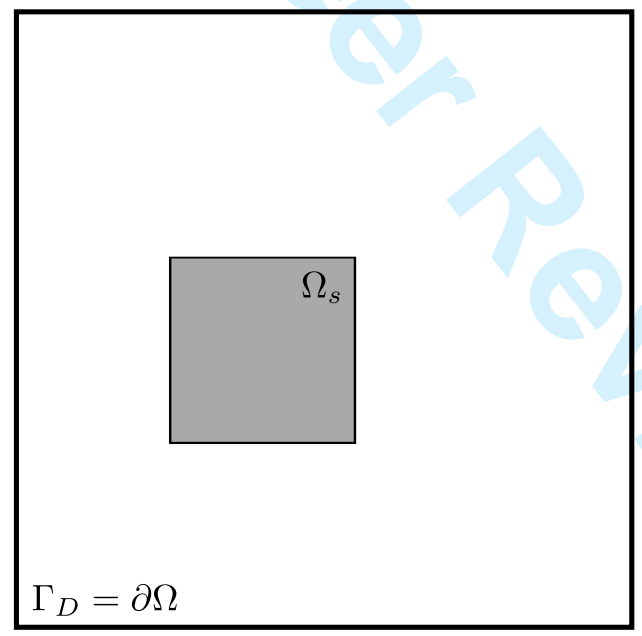

(a) Sketch of the domain

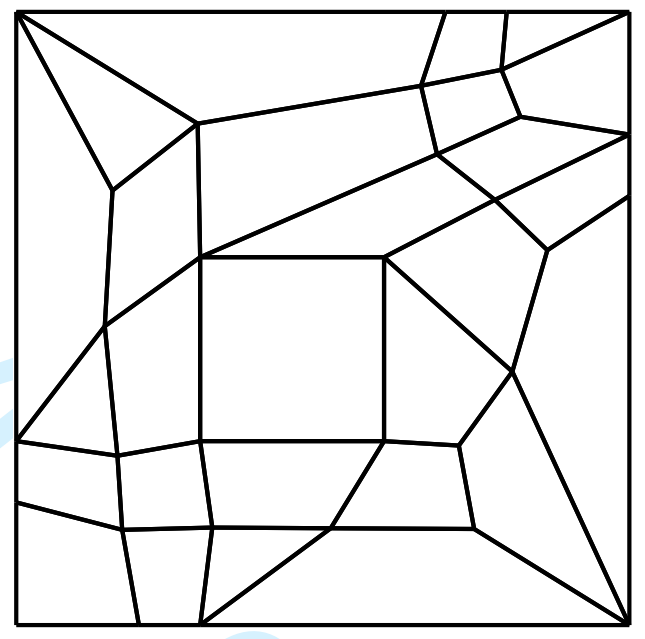

(b) Hybrid mesh of 27 elements

Figure 11. Anisotropic, non-homogeneous diffusion problem: system (1-2) coupled with (33).

The anisotropic and non-homogeneous diffusivity tensor $\mathbf{D}$ is defined as

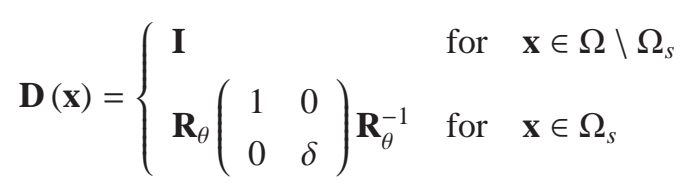

where $\mathbf{R}_{\theta}$ denotes the rotation matrix with $\theta=10^{\circ}$ and $\delta=10^{-3}$.

Figure 12 shows the solution by the proposed DCVFEM. Additionally, the problem with uniform diffusivity is solved for reference, as shown in Figure 12a, whereas Figure 12b shows the solution with anisotropic and discontinuous coefficients as in (34). A non-uniform polynomial order is used such that $P^{e}=4$ in the single element in the inclusion $\Omega_{s}$, while $P^{e}=2$ is set in the rest of the domain. The contour of the scalar $u$ is plotted and streamlines of the vector quantity $\mathbf{q}$ are used to visualize the deviation of the flow in the inclusion $\Omega_{s}$. 


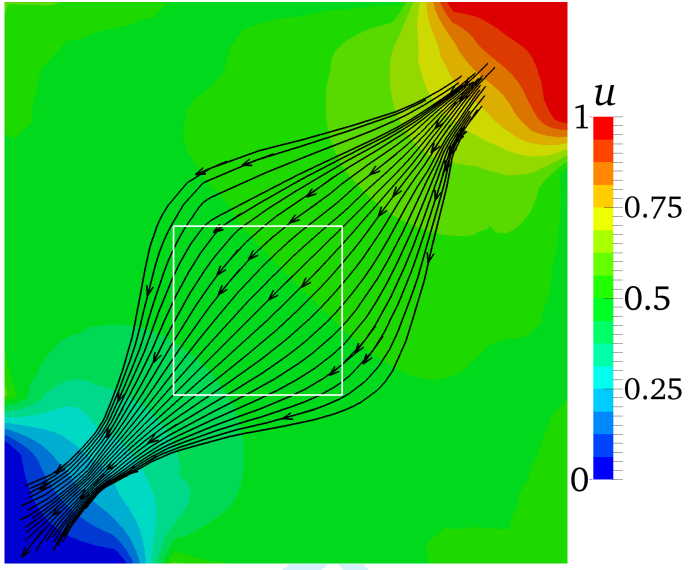

(a) Homogeneous and isotropic case $(\delta=1)$

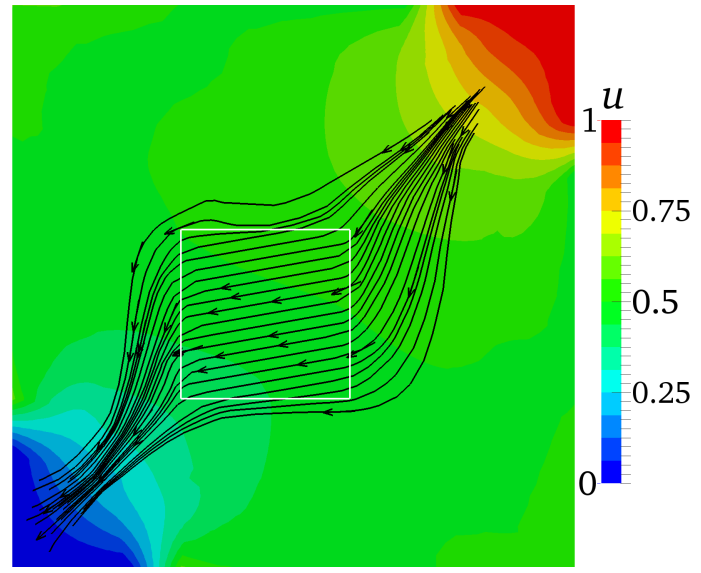

(b) Inhomogeneous and anisotropic case $\left(\delta=10^{-3}\right)$

Figure 12. Solution to system (1-2) coupled with (33): distribution of the scalar $u$ and streamlines of the flux q.

The DCVFEM can be successfully used to solve problems with anisotropic diffusion coefficients on hybrid meshes. The discontinuous approach allows to increase or decrease locally the polynomial order of accuracy, for enhanced resolution or saving computational cost.

\section{CONCLUDING REMARKS}

Quadrilateral and triangular elements with curvilinear edges and arbitrary polynomial order are developed for the two-dimensional discontinuous control-volume finite-element method (DCVFEM). A formal description of the elemental mapping by the blending method is provided. The piecewise-polynomial functions used for both the elemental mapping and the approximation of the unknowns belong to the same finite-dimensional space. A single assembly procedure accommodates all types of elements, resulting in a straight forward application to hybrid meshes. The expected spectral convergence of the method in the presence of curved boundaries is confirmed numerically both on triangular and on hybrid meshes. The method is successfully applied to the solution of problems with discontinuous and anisotropic diffusivity.

\section{ACKNOWLEDGEMENT}

The authors gratefully acknowledge the financial support of Diputación Foral de Bizkaia (grant BFA/DFB6/12/TK/2012/00020).

\section{REFERENCES}

1. Martinez MJ. Comparison of Galerkin and control volume finite element for advection-diffusion problems. International Journal for Numerical Methods in Fluids 2006; 50:347-376.

2. Baliga BR, Patankar SV. A new finite-element formulation for convection-diffusion problems. Numerical Heat Transfer, Part B: Fundamentals 1980; 3(4):393-409. 
3. Ramadhyani S, Patankar SV. Solution of the poisson equation: Comparison of the galerkin and control-volume methods. International Journal for Numerical Methods in Engineering 1980; 15(9):1395-1402.

4. Ramadhyani S, Patankar SV. Solution of the convection-diffusion equation by a finite-element method using quadrilateral elements. Numerical Heat Transfer Part B-fundamentals 1985; 8:595-612.

5. Schneider GE, Raw MJ. A skewed, positive influence coefficient upwinding procedure for control-volume-based finite-element convection-diffusion computation. Numerical Heat Transfer, Part B: Fundamentals 1986; 9(1):1-26.

6. Masson C, Saabas HJ, Baliga BR. Co-located equal-order control-volume finite element method for twodimensional axisymmetric incompressible fluid flow. International Journal for Numerical Methods in Fluids 1994; 18:1-26.

7. Saabas HJ, Baliga BR. Co-located equal-order control-volume finite-element method for multidimensional, incompressible fluid flow-part I: formulation. Numerical Heat Transfer, Part B: Fundamentals 1994; 26(4):381407.

8. Saabas HJ, Baliga BR. Co-located equal-order control-volume finite-element method for multidimensional, incompressible fluid flow-part II: verification. Numerical Heat Transfer, Part B: Fundamentals 1994; 26(4):409424.

9. Banaszek J. Comparison of control volume and Galerkin finite element methods for diffusion-type problems. Numerical Heat Transfer, Part B: Fundamentals 1989; 16(1):59-78.

10. Ciarlet PC. Discrete maximum principle for finite difference operators. Aequationes Mathematicae 2000; 38:16761706.

11. Piller M, Stalio E. Development of a mixed control volume - finite element method for the advection-diffusion equation with spectral convergence. Computers $\mathcal{E}$ Fluids 2011; 40(1):269-279.

12. Cockburn B. Discontinuous Galerkin methods. Journal of Applied Mathematics and Mechanics 2003; 11:731-754.

13. Li BQ. Discontinuous finite elements in fluid dynamics and heat transfer. Springer-Verlag, Berlin, 2006.

14. Choi BJ, Iskandarani M, Levin JC, Haidvogel DB. A spectral finite volume method for the shallow water equation. Monthly Weather Review 2004; 132(7):1777-1791.

15. Iskandarani M, Levin JC, Choi BJ, Haidvogel DB. Comparison of advection schemes for high-order h-p finite element and finite volume methods. Ocean Modeling 2004; 10:233-252.

16. Wang ZJ. Spectral (finite) volume method for conservation laws on unstructured grids: basic formulation. Journal of Computational Physics 2002; 178:210-251.

17. Wang ZJ, Liu Y. Spectral (finite) volume method for conservation laws on unstructured grids. II: extension to two-dimensional scalar equation. Journal of Computational Physics 2002; 179:665.

18. Wang ZJ, Liu Y. Spectral (finite) volume method for conservation laws on unstructured grids. III: extension to one-dimensional systems. Journal of Scientific Computing 2004; 20:137.

19. Wang ZJ, Liu Y. Spectral (finite) volume method for conservation laws on unstructured grids. IV: extension to two-dimensional Euler equations. Journal of Computational Physics 2004; 194:716.

20. Wang ZJ, Liu Y. Extension of the spectral volume method to high-order boundary representation. Journal of Computational Physics 2006; 211:154-178.

21. Liu Y, Vinokur M, Wang ZJ. Spectral (finite) volume method for conservation laws on unstructured grids V: Extension to three-dimensional systems. Journal of Computational Physics March 2006; 212:454-472.

22. Kannan R, Wang ZJ. The direct discontinuous Galerkin (DDG) viscous flux scheme for the high order spectral volume method. Computers $\mathcal{E}$ Fluids 2010; 39:2007-2021.

23. Stipcich G, Piller M, Pivetta M, Zovatto L. Discontinuous control-volume/finite-element method for advectiondiffusion problems. Computers $\mathcal{E}$ Fluids 2011; 52(0):33-49.

24. Zienkiewicz OC, Taylor RL. The finite element method. Volume 1: The basis. Butterworth-Heinemann, Oxford, 2000.

25. Fortin M, Brezzi F. Mixed and hybrid finite element methods. Springer-Verlag, Berlin, 1991.

26. Cockburn B, Shu CW. The local discontinuous Galerkin method for time-dependent convection-diffusion systems. SIAM Journal on Numerical Analysis 1998; 35:2440-2463.

27. Sherwin SJ, Karniadakis GE. Tetrahedral hp finite elements: algorithms and flow simulations. Journal of Computational Physics 1996; 124(1):14-45.

28. Szabó B, Babuška I. Finite element analysis. Springer-Verlag, Berlin, 1991.

29. Onsager L. Reciprocal relations in irreversible processes. i. Phys. Rev. Feb 1931; 37:405-426, doi:10.1103/PhysRev. 37.405. URL http://link. aps.org/doi/10.1103/PhysRev. 37.405.

30. Quarteroni A, Valli A. Numerical approximation of partial differential equations. Springer-Verlag Italia, Milano, 1994.

31. Karniadakis GE, Sherwin SJ. Spectral/hp element methods for CFD. Oxford University Press: Oxford, 2005. 
32. Gordon WJ. Blending-function methods of bivariate and multivariate interpolation and approximation. SIAM Journal on Numerical Analysis 1971; 8(1):158-177.

33. Gordon WJ, Hall CA. Construction of curvilinear co-ordinate systems and applications to mesh generation. International Journal for Numerical Methods in Engineering 1973; 7(4):461-477, doi:10.1002/nme.1620070405. URL http: //dx . doi . org/10.1002/nme. 1620070405.

34. Gordon WJ, Hall CA. Transfinite element methods: Blending-function interpolation over arbitrary curved element domains. Numerische Mathematik 1973; 21(2):109-129, doi:10.1007/BF01436298. URL http://dx.doi.org/10.1007/BF01436298.

35. Duffy MG. Quadrature over a pyramid or cube of integrands with a singularity at a vertex. SIAM Journal on Numerical Analysis 1982; 19(6):1260-1262, doi:10.1007/BF01436298. URL http: //www. jstor .org/stable/2157208.

36. Bathe KJ. Finite element procedures. Prentice Hall, New Jersey, 1996.

37. Castillo P, Cockburn B, Perugia I, Schötzau D. An a priori error analysis of the local discontinuous Galerkin method for elliptic problems. SIAM Journal on Numerical Analysis 2000; 38:1676-1706.

38. Chidyagwai P, Mishev I, Rivière B. On the coupling of finite volume and discontinuous galerkin method for elliptic problems. Journal of Computational and Applied Mathematics 2011; 235(8):2193-2204, doi:http://dx.doi.org/10.1016/j.cam.2010.10.017. URL http://www. sciencedirect.com/science/article/pii/S0377042710005832.

39. Herbin R, Hubert F. Benchmark on Discretization Schemes for Anisotropic Diffusion Problems on General Grids. Finite volumes for complex applications V, ISTE (ed.), Wiley: France, 2008; 659-692. URL http://hal . archives-ouvertes. fr/hal-00429843. 